\title{
Research on Vibration of Wind-Power Tower Based on Response Spectrum Analysis
}

\author{
Mingliang YANG*, Wenjun MENG**, Zhengyan CHANG***, ShouchengWANG****, \\ Peng WANG***** \\ *School of Machinery and Electronics Engineering, Taiyuan University of Science and Technology, Taiyuan, Shanxi, \\ 030024, China, E-mail: yangmingliang1997@163.com \\ **School of Machinery and Electronics Engineering, Taiyuan University of Science and Technology, Taiyuan, Shanxi, \\ 030024, China, E-mail: tyustmwj@126.com \\ ***School of Machinery and Electronics Engineering, Taiyuan University of Science and Technology, Taiyuan, Shanxi, \\ 030024, China,E-mail: 535061752@qq.com \\ ****Taiyuan Heavy Industry Co., Ltd, Taiyuan, Shanxi, 030000, China, E-mail: 13603535316@139.com \\ *****School of Machinery and Electronics Engineering, Taiyuan University of Science and Technology, Taiyuan, Shanxi, \\ 030024, China, E-mail: 595855655 @qq.com
}

cross $^{\text {ref }}$ http://dx.doi.org/10.5755/j01.mech.23.6.19852

\section{Introduction}

The wind-power tower is a flexible structure the characteristics of 'high, large, long and thin'. The width and depth of the tower is much smaller than its height. The wind load is one of the main design loads, which is divided into two parts, including static wind load and fluctuating wind load. The latter can cause structural vibration [1]. For the calculation of wind-induced vibration, the peak response of the structure considered as a linear structure is concerned and the spectral analysis method is used to solve the problem [2-4]. Based on the analysis of wind load response, the response of the structure with dampers or without dampers is analyzed by the spectrum analysis method.

\section{Transverse wind load response}

Under the action of horizontal wind, the tower structure with circular cross section will only cause the lateral wind response. When the airflow rounds the tower structure, a vortex will be formed due to the airflow meeting again behind the structure. So, the maximum acceleration of the structure may occur in the crosswind.

After the air flows tower, flow characteristics at different stages are divided into three ranges according to the Reynolds number [1], as shown in Fig. 1.

Subcritical range is $3 \times 10^{2}<\operatorname{Re}<3 \times 10^{5}$. and the periodic vibration of structure is caused by the periodic vortex shedding.

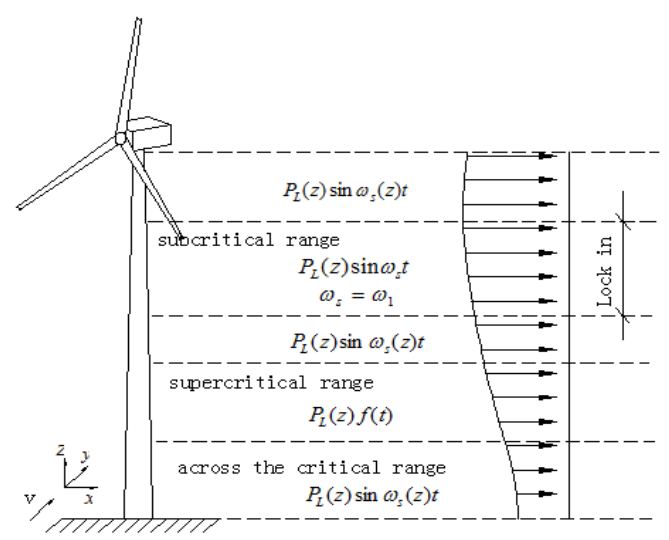

Fig. 1 Schematic diagram of transverse wind partition
Supper critical ranges $3 \times 10^{5} \leq R e<3.5 \times 10^{6}$ and the random vibration will be produced for structure, irregularly.

Trans critical range is $3 \times 10^{5} \leq \operatorname{Re}<3.5 \times 10^{6}$ and the deterministic vibration will be occurred periodically.

Experiments show that when the vortex shedding frequency reaches the natural frequencies of the structure owing to the increase of wind speed, the frequency is constant in the wind speed region, which is called the locked area. Especially when the vortex shedding frequency is consistent with the natural frequency of the structure, a large resonance response will be generated.

Reynolds number formula is expressed as:

$$
R_{e}=\frac{v l}{\gamma}
$$

Where: $v$ is the wind speed. $l$ is the maximum size of the cross-section of wind-power tower. $\gamma$ is the dynamic viscosity. For the air, it is $0.145 \times 10^{-4} \mathrm{~m}^{2} / \mathrm{s}$.

$R e=69000 \mathrm{vl}$.

And etc.

From Eq. (2), it can be seen that the Reynolds number is proportional to the wind speed. Therefore, the key of the wind power structure design is to check whether the resonance wind speed is a critical cross range or not. Especially when the frequency of vortex shedding is consistent with the structure frequency, it will produce a resonant response of several times larger than that of the static force [1]. For the structure in the sub critical range, although it can also occur resonance, the wind speed is smaller. For the structure function being not as serious as the cross critical range, the method of increasing structural stiffness and strength is usually adopted to deal with it.

The vibration period of the wind-power tower is between 0.5 and $2.5 \mathrm{~s}$, and the resonance speed is from $40 \mathrm{~m} / \mathrm{s}$ to $24 \mathrm{~m} / \mathrm{s}$. For $R e>3.5 \times 10^{6}$, the wind speed can be in the extreme weather condition.

\section{Vibration control of wind-power tower structure}

The purpose of vibration control of wind-power tower is to reduce the displacement and acceleration response of the structure [5-10]. 


\subsection{Mechanical model of damper}

A high-rise structure in the $\mathrm{j}$ mass of the structure is installed with a tuned mass damper (TMD). For the structure under the action of the damper, the calculation diagram of the damper and tower structure is given in Fig. 2.

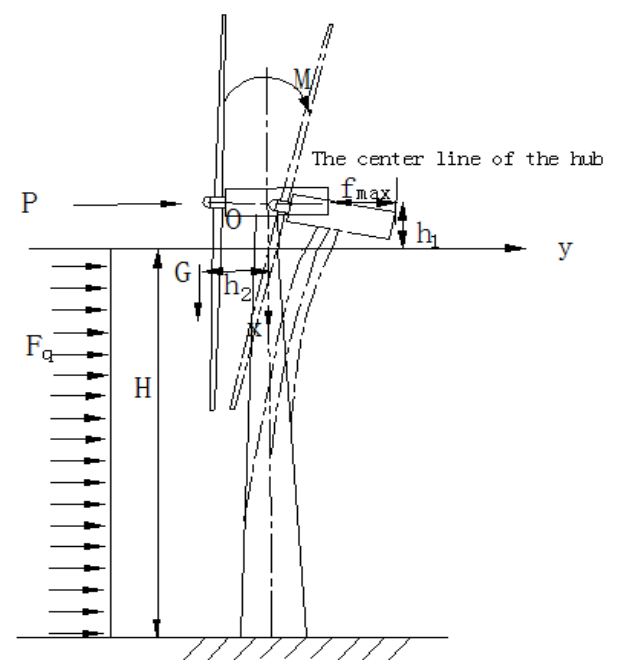

Fig. 2 Mechanical model of wind turbine tower

3.1.1. Motion equation of tower structure under TMD action

The motion equation of the tower structure under the TMD action can be designed as:

$$
[M]\{\ddot{x}\}+[C]\{\dot{x}\}+[K]\{x\}=\{f(z, t)\}+\left\{F_{T M D}\right\},
$$

where: $[M],[C]$ and $[K]$ are the mass, damping and stiffness matrixes of the tower structure, respectively. $\{\ddot{x}\}\{\dot{x}\}\{x\}$ are the acceleration, velocity and displacement vectors of the tower structure with respect to the ground, respectively. $\{f(z, t)\}$ is the wind load vector. $\left\{f_{T M D}\right\}$ is the damping load vector.

$$
\left\{F_{T M D}\right\}=\left[\begin{array}{c}
0 \\
\vdots \\
0 \\
c_{d}\left(\dot{x}_{d}-\dot{x}_{j}\right)+k_{d}\left(x_{d}-x_{j}\right) \\
0 \\
\vdots \\
0
\end{array}\right] .
$$

\subsubsection{TMD motion equation}

The TMD motion equation is defined as:

$$
m_{d} \ddot{x}_{d}+c_{d}\left(\dot{x}_{d}-\dot{x}_{j}\right)+k_{d}\left(x_{d}-x_{j}\right)=0,
$$

TMD, respectively. $x_{j} \mathrm{~s}$ the displacement of the $j$ mass of the structure with respect to the ground. $x_{d}$ is the displacement of TMD relative to the ground.

\subsubsection{Mean square value of relative displacement of structure at any} point and TMD

After installing TMD, if only the first vibration mode is considered, Eq. (6) can be obtained by the Eq. (3) and $F_{1}=e^{i \omega t}$.

$$
\ddot{q}+2 \xi_{1} \omega_{1} \dot{q}+\omega_{1}^{2} q=\frac{\varphi_{j}}{M_{1}}\left(c_{d} \dot{v}+k_{d} v\right)+\frac{\{\varphi\}^{T}\{f(z, t)\}}{M_{1}},
$$

where: $v$ is the displacement of TMD with respect to the $j$ mass, $v=x_{d}-x_{j} . \mu$ is the ration of TMD mass and structure generalized mass, $\mu=m_{d} / M_{1} \cdot \zeta_{d}$ is the damping ratio of TMD, $\zeta_{d}=\frac{c_{d}}{2 m_{d} \omega_{d}} \cdot \omega_{d}$ is The natural frequency of TMD, $\omega_{d}=\sqrt{k_{d} / m_{d}} \cdot M_{1}$ is the generalized mass. $\zeta_{1}$ is the damping ratio of first modal for the structure. $\omega_{1}$ is the first natural frequency of structure. $\phi_{j}$ is the value of First mode at the point $\mathrm{j}$.

It can be obtained by Eq. (5). So,

$$
\begin{aligned}
& \ddot{q}+2 \zeta_{1} \omega_{1} \dot{q}+\omega_{1}^{2} q-\mu \varphi_{j}\left(2 \zeta_{d} \omega_{d} \dot{v}+\omega_{d}^{2} v\right)=F_{1}(t), \\
& \varphi_{j} \ddot{q}+\ddot{v}+2 \xi_{d} \omega_{d} \dot{v}+\omega_{d}^{2} v=0 .
\end{aligned}
$$

It can be obtained by bringing Eq. (8) into Eq. (7).

$$
\left(1+\mu \varphi_{j}^{2}\right) \ddot{q}+\mu \varphi_{j} \ddot{v}+2 \xi_{1} \omega_{1} \dot{q}+\omega_{1}^{2} q=F_{1}(t) .
$$

The formula (8) and type (9) are written in matrix form:

$$
\begin{gathered}
{\left[\begin{array}{cc}
1+\mu \varphi_{j}^{2} & \mu \varphi_{j} \\
\varphi_{j} & 1
\end{array}\right]\left\{\begin{array}{l}
\ddot{q} \\
\ddot{v}
\end{array}\right\}+\left[\begin{array}{cc}
2 \xi_{1} \omega_{1} & 0 \\
0 & 2 \xi_{d} \omega_{d}
\end{array}\right]\left\{\begin{array}{l}
\dot{q} \\
\dot{v}
\end{array}\right\}+} \\
+\left[\begin{array}{cc}
\omega_{1}^{2} & 0 \\
0 & \omega_{d}^{2}
\end{array}\right]\left\{\begin{array}{c}
F_{1}(t) \\
0
\end{array}\right\}, \\
F_{1}(t)=e^{i \omega t}, \text { so } \\
\left\{\begin{array}{l}
q \\
v
\end{array}\right\}=\left\{\begin{array}{l}
H_{q}(\omega) \\
H_{v}(\omega)
\end{array}\right\} e^{i \omega t} .
\end{gathered}
$$

Eq. (11) can be simplified as:

where: $m_{d}, c_{d}$ and $k_{d}$ are the mass, damping and spring stiffness of

$$
\left\{\begin{array}{cc}
-\left(1+\mu \varphi_{j}^{2}\right) \omega^{2}+2 \zeta_{1} \omega_{1} i \omega+\omega_{1}^{2} & -\mu \varphi_{j}^{2} \omega^{2} \\
-\varphi_{j} \omega^{2} & -\omega^{2}+2 \zeta_{d} \omega_{d} i \omega+\omega_{d}^{2}
\end{array}\right\} \cdot\left\{\begin{array}{l}
H_{q} \\
H_{v}
\end{array}\right\}=\left\{\begin{array}{l}
1 \\
0
\end{array}\right\} .
$$


Frequency response function matrix can be introduced as:

$$
\left\{\begin{array}{l}
H_{q} \\
H_{v}
\end{array}\right\}=\frac{1}{D}\left\{\begin{array}{cc}
-\omega^{2}+2 \zeta_{d} \omega_{d} i \omega+\omega_{d}^{2} & \mu \phi_{j}^{2} \omega^{2} \\
\phi_{j} \omega^{2} & -\left(1+\mu \phi_{j}^{2}\right) \omega^{2}+2 \zeta_{1} \omega_{1} i \omega+\omega_{1}^{2}
\end{array}\right\} \cdot\left\{\begin{array}{l}
1 \\
0
\end{array}\right\}
$$

Where:

$D=\omega^{4}-2\left(\zeta_{1} \omega_{1}+\zeta_{d} \omega_{d}+\mu \varphi_{j}^{2} \zeta_{d} \omega_{d}\right) \cdot i \omega^{3}-\left(\omega_{1}^{2}+\omega_{d}^{2}+\mu \varphi_{j}^{2} \zeta_{d}^{2} \omega_{d}^{2}+4 \zeta_{1} \zeta_{d} \omega_{d} \omega_{1}\right) \omega^{2}+2\left(\zeta_{1} \omega_{1} \omega_{d}^{2}+\zeta_{d} \omega_{d} \omega_{1}^{2}\right) \cdot i \omega+\omega_{1}^{2} \omega_{d}^{2}$.

The spectral density function of stochastic generalized coordinates is defined as:

$$
\begin{aligned}
& S_{q}=H_{q}(\omega) S_{F 1}(\omega) H_{q}^{*}(\omega)=\left|H_{q}(\omega)\right|^{2} S_{F_{1}}(\omega), \\
& S_{F 1}(\omega)=\frac{\{\varphi\}^{T}\left[S_{P}(\omega)\right]\{\varphi\}}{M_{1}^{2}}, \\
& S_{F}=\left\{\begin{array}{c}
\varphi_{1}^{2} \\
\vdots \\
\varphi_{n}^{2}
\end{array}\right\} \cdot\left|H_{q}(\omega)^{2}\right| S_{F_{1}}(\omega) .
\end{aligned}
$$

When the TMD is installed, the mean square value of the displacement of the structure at any point $m$ can be designed as:

$$
\begin{aligned}
& \sigma_{x^{m} T M D}^{2}=\int_{-\infty}^{+\infty} S_{x^{m}} d \omega= \\
& =\frac{\varphi_{m}^{2} \sum_{i=1}^{n} \sum_{j=1}^{m} \varphi_{i} \varphi_{j} \rho_{i j} p_{i} p_{j}}{\left(\sum_{i=1}^{n} m_{i} \varphi_{i}^{2}\right)^{2}} \int_{-\infty}^{+\infty}\left|H_{q}(\omega)\right|^{2} S_{f}(\omega) d \omega .
\end{aligned}
$$

After the installation of TMD, with no TMD, of the ratio of the displacement mean square value of the structure at any point $m$ is given as:

$$
\begin{gathered}
\sigma_{n o r m m}^{2}=\frac{\sigma_{x^{m} T M D}^{2}}{\sigma_{x^{m}}^{2}}=\frac{\int_{-\infty}^{+\infty}\left|H_{q}(\omega)\right|^{2} S_{f}(\omega) d \omega}{\int_{-\infty}^{+\infty}\left|H_{1}(\omega)\right|^{2} S_{f}(\omega) d \omega} . \quad \text { (19) } \begin{array}{r}
\text { According to the integ } \\
\text { frequency response function, }
\end{array} \\
\int_{-\infty}^{+\infty}\left|H_{q}(\omega)\right|^{2} d \omega=\frac{\pi}{2 \omega_{1}^{3}} \cdot \frac{2 A_{1} \zeta_{d} \lambda^{4}+2 B_{1} \zeta_{1} \lambda^{3}+\left[4 C_{1}-2 \mu \varphi_{J}^{2}\right] \zeta_{d} \lambda^{2}+8 \zeta_{1} \zeta_{d}^{2} \lambda+2 \zeta_{d}}{4 A_{1} \zeta_{d} \lambda^{4}+4 B_{1} \zeta_{1}^{2} \lambda^{3}+8 C_{1} \zeta_{1} \zeta_{d}^{2} \lambda^{2}+4\left(\mu \varphi_{J}^{2}+4 \zeta_{1}^{2}\right) \zeta_{d}^{2} \lambda+4 \zeta_{1} \zeta_{d}} .
\end{gathered}
$$

Similarly, the power spectral density $S_{v}(\omega)$ of the relative displacement of the TMD mass can be obtained as:

$$
S_{v}=\left|H_{v}(\omega)\right|^{2} S_{F_{1}}(\omega)
$$

The mean square value of the relative displacement of TMD can be expressed as:

$$
\begin{aligned}
& \sigma_{V}^{2}=\int_{-\infty}^{+\infty} S_{v}(\omega) d \omega= \\
& =\frac{\sum_{i=1}^{n} \sum_{j=1}^{m} \varphi_{i} \varphi_{j} \rho_{i j} p_{i} p_{j}}{\left(\sum_{i=1}^{n} m_{i} \varphi_{i}^{2}\right)^{2}} \int_{-\infty}^{+\infty}\left|H_{v}(\omega)\right|^{2} S_{f}(\omega) d \omega .
\end{aligned}
$$

\subsection{Structural damper}

The damping device can reduce the effect of the wind vibration response, which is caused by the shortening of the system period due to the additional stiffness of dampers and the reduction of the energy generated by the damping characteristic of the damper [11].

In order to analyze the influence of the parameters of TMD on the wind load of tower structure, for tower structure with and without TMD, the displacement formula of the wind load is expressed by the parameters of TMD, mass, damping and stiffness.

\subsubsection{Frequency response function}

According to the integral formula of the complex

Where: $\lambda=\omega_{d} / \omega_{1} ; \mu=m_{d} / M_{1} ; A_{1}=\left(1+\mu \varphi_{J}^{2}\right)^{2} ; B_{1}=\mu \varphi_{J}^{2}+4\left(1+\mu \varphi_{J}^{2}\right) \zeta_{d}^{2} ; C_{1}=2 \zeta_{1}^{2}+2\left(1+\mu \varphi_{J}^{2}\right) \zeta_{d}^{2}-1$.

Let,

$$
C\left(\mu, \zeta_{d}, \lambda\right)=\frac{2 A_{1} \zeta_{d} \lambda^{4}+2 B_{1} \zeta_{1} \lambda^{3}+\left[4 C_{1}-2 \mu \varphi_{J}^{2}\right] \zeta_{d} \lambda^{2}+8 \zeta_{1} \zeta_{d}^{2} \lambda+2 \zeta_{d}}{4 A_{1} \zeta_{d} \lambda^{4}+4 B_{1} \zeta_{1}^{2} \lambda^{3}+8 C_{1} \zeta_{1} \zeta_{d}^{2} \lambda^{2}+4\left(\mu \varphi_{J}^{2}+4 \zeta_{1}^{2}\right) \zeta_{d}^{2} \lambda+4 \zeta_{1} \zeta_{d}}
$$

So, 


$$
\begin{aligned}
& H S_{2}=\int_{-\infty}^{+\infty}\left|H_{q}(\omega)\right|^{2} S_{f}(\omega) d \omega= \\
& =\frac{1}{\omega_{1}^{4}}+\frac{\pi}{2 \omega_{1}^{3}} C\left(\mu, \zeta_{d}, \lambda\right) S_{f}\left(\omega_{1}\right) \text {. } \\
& \zeta_{e}=\frac{1}{2 C\left(\mu, \zeta_{d}, \lambda\right)}=\zeta_{1}+\frac{\mu \varphi_{j}^{2} \zeta_{d} \lambda\left(\zeta_{d}+\zeta_{1} \lambda\right)}{A_{1} \zeta_{d} \lambda^{4}+B_{1} \zeta_{1} \lambda^{3}+\left[2 C_{1}-\mu \varphi_{j}^{2}\right] \zeta_{d} \lambda^{2}+4 \zeta_{1} \zeta_{d}^{2} \lambda+\zeta_{d}}
\end{aligned}
$$

\subsubsection{Equivalent damping}

The equivalent damping of the structure with TMD
From Eq. (25), it can be seen that when the structure of TMD is set up, the damping of the tower structure will be increased. The greater the damping, the smaller the response is. The equivalent damping is related to the mass $m_{d}$, damping ratio $\zeta_{d}$ and stiffness $k_{d}$ of TMD and it is also related to the position of TMD in the structure.

\subsubsection{Parameters selection}

The frequency modulation quality controller is composed of four parts: mass block, spring system, damping system and mass block supporting system, as shown in Fig. 3.

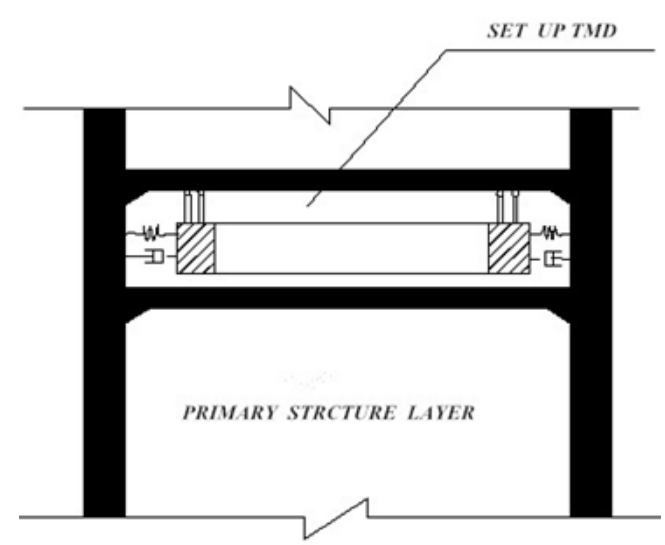

Fig. 3 Pendant frequency modulation mass damper of tower tube of wind generator

In the vibration control. The inertia force generated by the mass block and the relative motion of the structure is mainly used to achieve the purpose of reducing vibration. The ratio of the mass and the tower structure quality is between 0.02 and 0.005 [4].

The spring system is composed of 4 helical springs. The function is to adjust the frequency of the vibration control, so that it is close to the controlled natural frequency of the structure.

The damping system provides damping for the whole vibration control to achieve the good vibration absorbing effect. The appropriate damping coefficient is chosen from $1 \times 10^{6} \mathrm{Ns} / \mathrm{m}$ to $12 \times 10^{6} \mathrm{Ns} / \mathrm{m}$.

The mass support system is a hanging hook and the friction between the hook and the mass is very small, which can ensure free sloshing of the mass block.

\section{Response spectrum analysis}

The response spectrum analysis is mainly used to determine the dynamic response of wind-power tower structure to random wind load. The result of tower modal analysis is connected with the wind speed spectrum to calculate the displacement and stress of the model.

\subsection{Response spectrum}

Random vibration of wind power tower under dynamic wind load will be produced and the stochastic process simulation should be used in dynamic response analysis. In this paper, the linear filtering method combined with Matlab software is used to simulate the fluctuating wind speed time history of the wind-power tower under the wind load.

Wind speed spectrum for structural analysis davenport spectrum [12].

$$
\left\{\begin{array}{c}
S_{v(n)}=4 k v_{10}^{-2} \frac{x^{2}}{n\left(1+x^{2}\right)^{4 / 3}} . \\
x=1200 n \sqrt{v_{10}}
\end{array}\right.
$$

Where: $n$ is the frequency of wind speed in the wind, Hz. $x$ is the turbulence integral scale coefficient. $\bar{v}_{10}$ is the average wind speed at $10 \mathrm{~m}$ height from the ground. $k$ is the surface drag coefficient, Corresponding to the values of $A, B, C$, and $D$. Four type landforms for $k$ are shown in Table 1.

Table 1

Four type landforms for $k$

\begin{tabular}{|c|c|c|c|c|}
\hline $\begin{array}{c}\text { Land- } \\
\text { forms }\end{array}$ & $A$ & $B$ & $C$ & $D$ \\
\hline$k$ & 0.00129 & 0.00215 & 0.00464 & 0.01291 \\
\hline
\end{tabular}

For the wind speed being $11.4 \mathrm{~m} / \mathrm{s}$ and wind-power generator being in the standard condition, the Reynolds number $R e$ is $2.934 \times 106$. The structure is not conducted the check calculation in the supercritical range, because the tower wind vortex shedding wind vibration is not formed, obviously.

For the wind speed being $25 \mathrm{~m} / \mathrm{s}$ and wind-power generator being in the cutting condition, the Reynolds number $R e$ is $6.43 \times 106$. At this time, the structure may occur the resonance exceeding the critical range. So, the resonance response should be calculated.

For the wind speed being $50 \mathrm{~m} / \mathrm{s}$ and wind turbines being in extreme condition, the Reynolds number $R e$ is $12.87 \times 106$. At this time, the structure exceeds the critical range. So, the resonance response should be determined. Using the linear filtering method, under the three conditions 
including standard operating condition, cutting out condition and limit condition, the fluctuating wind speed time history of wind-power tower structure bearing the wind load is simulated by Matlab. For the limit of speed being $50 \mathrm{~m} / \mathrm{s}$, the wind velocity spectrum is as shown in Fig. 4 and Fig. 5.

From the above chart, the power spectral density function curve of the numerical simulation is in good agreement with the target spectral density curve. So, the simulation curve of fluctuating wind speed- time history of wheel center has a very high accuracy. The wind speed spectrum curve can be adopted to conduct the finite element wind vibration response analysis to wind-power tower structure.

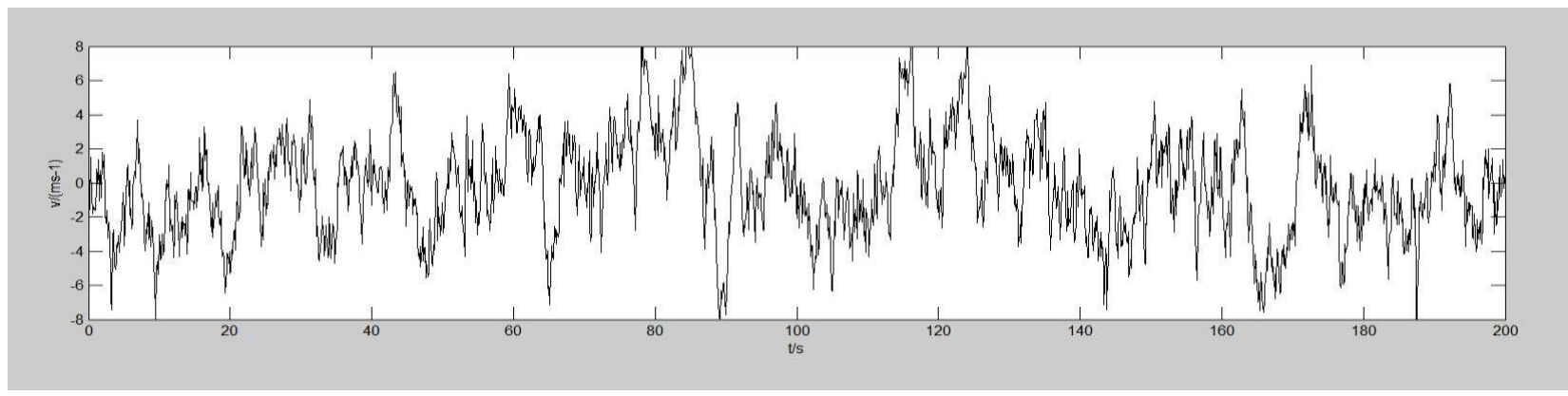

Fig. 4 Wind conditions the simulated wind speed spectrum of wheel center under $50 \mathrm{~m} / \mathrm{s}$ wind speed

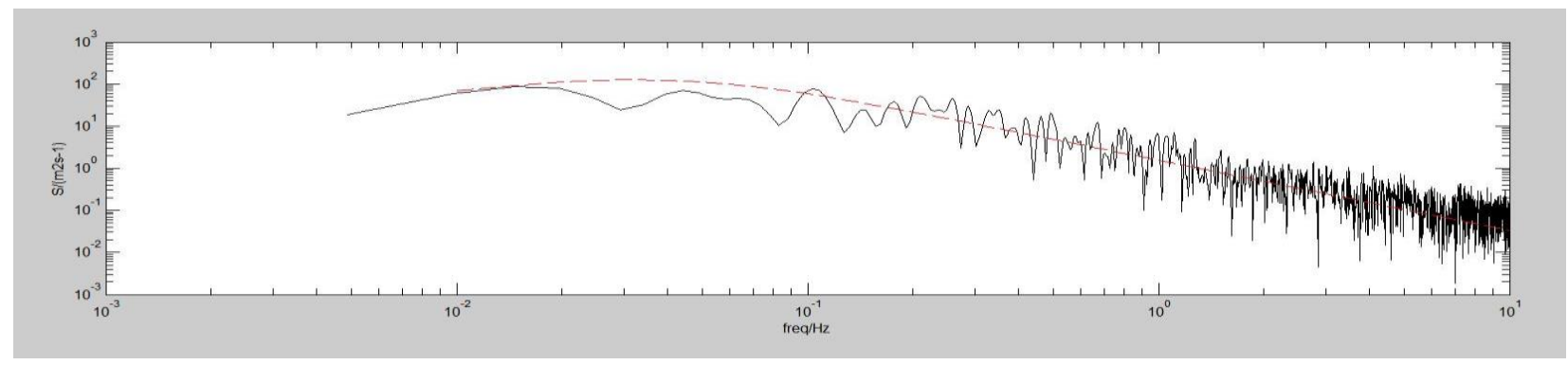

Fig. 5 Comparison of power spectrum and target spectrum for simulating fluctuating wind speed of wheelcenterunder $50 \mathrm{~m} / \mathrm{s}$ wind speed

\subsection{Analysis process}

Three dimensional model of the wind power tower structure is built with the shell element, $Q 345 E$ material and cantilever beam mechanical model. The numerical value is intro- duced into the finite element analysis software. The response of tower tube structure with damper or without damper is analyzed by the random vibration theory under three wind speed conditions. The calculation results are shown in Table 2.

Table 2

Tower structure response under limit conditions

\begin{tabular}{|c|c|c|c|c|}
\hline \multirow{2}{*}{ Wind speed $(\mathrm{m} / \mathrm{s})$} & \multicolumn{2}{|c|}{ Tower structure displacement $(\mathrm{mm})$} & \multicolumn{2}{c|}{ Tower structure acceleration $\left(\mathrm{m} / \mathrm{s}^{2}\right)$} \\
\cline { 2 - 5 } & Without damper & With Damper & Without damper & With Damper \\
\hline 11.4 & 769.7 & 147 & 2.791 & 1.26 \\
\hline 25 & 1564 & 748 & 9.076 & 3.85 \\
\hline 50 & 1282 & 692.3 & 15.87 & 4.45 \\
\hline
\end{tabular}

For the wind speed being $50 \mathrm{~m} / \mathrm{s}$, the displacement response of the structure is presented in Fig. 6.

It can be seen from Table 2 that the maximum displacement and acceleration of structures under extreme climatic conditions are greatly reduced for providing the suspension tuned mass damper.

\section{Conclusion}

In this paper, the response of wind power tower structure is studied by the spectral analysis method under three different conditions and the vibration control equipment is used to make the comparison of structures. There are the following main conclusions:

1) The AR method of the linear filtering method is used to simulate the wind speed spectrum of tower structure under three different conditions and the fluctuating wind speed simulation spectrum is always floating around the target spectrum curve and almost symmetrical, which indicate that wind speed meets expectations and has a fairly high accuracy. So, it can be used to analyze the wind-induced vibration response of the wind-power tower.

2) Three dimensional finite element analysis model of wind power tower is built, and the random vibration analysis of the structure is carried out. From the analysis results, for the wind speed being $11.4 \mathrm{~m} / \mathrm{s}$, the displacement and acceleration of the structure in the supercritical range is smaller and the tower wind vortex shedding wind vibration is not formed, obviously. For the wind speed being $25 \mathrm{~m} / \mathrm{s}$ and wind-power generator being in the cutting condition, the structure may occur the resonance exceeding the critical range. So, the resonance response should be calculated. For the wind speed being $50 \mathrm{~m} / \mathrm{s}$ and wind turbines being in extreme condition, the structure exceeds the critical range. At this time, the structure displacement and acceleration are 
larger, but the value is less than the cut off working condition.

3) The maximum displacement and acceleration of the structure are greatly reduced under three different wind loads, owing to the suspension tuned mass damper be set.

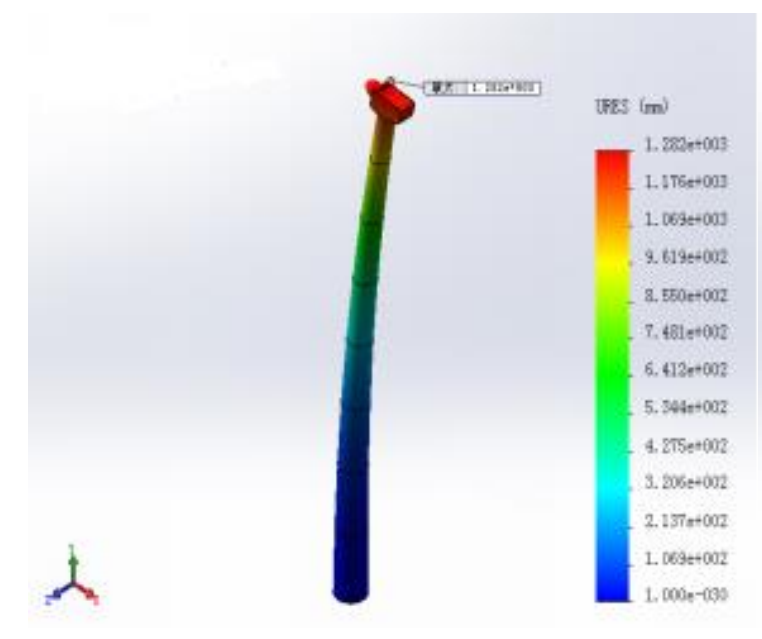

a. withdamper

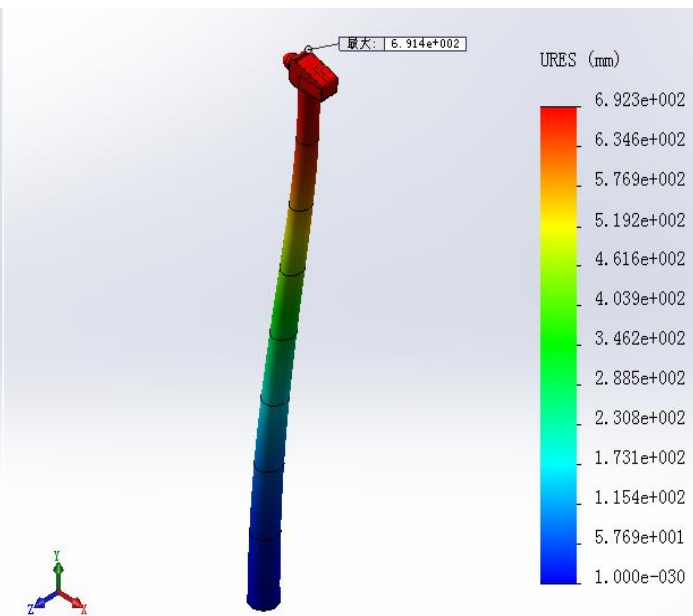

b. without damper

Fig. 6 Displacement of the tower structure under the $50 \mathrm{~m} / \mathrm{s}$ condition

\section{Acknowledgements}

This paper is supported by the Postdoctoral Startup Foundation of Taiyuan University of Science and Technology(No.20142011), the Doctor Startup Foundation of Taiyuan University of Science(No.20122001), the Fund for Shanxi "1331 project " Key Subjects Construction (1331KSC).

\section{References}

1. Zhang, X. T. 2013. Wind load theory of engineering structure and wind resistant calculation manual. Tongji University press: 56-59 p.

2. Zhang, P. X. Building structure and wind load. Shanghai science and Technology Publishing House: 109-113 p.

3. Qu, W. L.; Wei, W. H. 2010. Analysis and prevention of collapse process of disaster wind and wind-induced tall. Proceedings of The 30 Annual Conference on Structural Wind Engineering in China.

4. Wang, Z. M. 1994. Research and design of TMD wind vibration control of TV tower structure, Journal of building structures: $2-13 \mathrm{p}$.

http://dx.doi.org/10.14006/j.jzjgxb.1994.05.001.

5. Liu, Y.; Xu, X.; Zhou, X. J. 2009. Dynamic response and wind vibration control of high-rise structures under the action of typhoon. Noise and Vibration Control: 30$34 \mathrm{p}$.

http://dx.doi.org/10.3969/j.issn.1006-1355.2009.02.009.

6. Feng, Y. Q.; Chen, J. L. 2011. The acceleration input equivalent wind turbine tower measured response method. Journal of Shijiazhuang Tiedao University: Science Edition: 21-24 p.

7. Luo, Ch.; Cao, W.S. The impact of typhoon on the development of china's offshore wind power. Energy and Environment: $2-3 \mathrm{p}$. http://dx.doi.org/10.3969/j.issn.16729064.2013.03.001.
8. Guang, L.; Tian, J.K.; Chang, D.S. 2013. Offshore wind turbine anti typhoon concept design. Electric Power Construction: 11-17 p. http://dx.doi.org/10.3969/j.issn.10007229.2013.02.003.

9. Wu, J. C.; Zhang, R. Y.; Zhang, X. Z. 2010. Anti-typhoon design of offshore wind turbines. China Engineering Science: $25-31 \mathrm{p}$. http://dx.doi.org/10.3969/j.issn.10091742.2010.11.005.

10. Prowell, I.; Veletzos, M.; Elgamal, A. 2009. Experimental and numerical scismic response of a $65 \mathrm{kw}$ wind turbine steel tower. Journal of Earthquake Engineering:1172 p.

https://doi.org/10.1080/13632460902898324.

11. Lu, W.D.; Liu, W.Q.; Wang, T. 2009. Calculation method for additional equivalent damping ratio of energy dissipation structure. Journal of of Nanjing University of Technology: 97-100 p. http://dx.doi.org/10.3969/j.issn.16717627.2009.01.017.

12. Wang, X.Q.; Cui, J.F.. 2002. Formula of coefficient K in expression of davenport spectrum and its engineering applicationJournal of Tongji University: 849-851 p. http://dx.doi.org/10.3321/j.issn:0253374X.2002.07.015. 
Mingliang Yang, Wenjun Meng, Zhengyan Chang, Shoucheng Wang, PengWang

\section{RESEARCH ON VIBRATION OF WIND-POWER TOWER BASED ON RESPONSE SPECTRUM} ANALYSIS

\section{S u m m a r y}

As a flexible structure with the height-to-width ratio being quite large, the wind-power tower structure is easily produce the wind-induced vibration under the action of dynamic wind load. First of all, the probabilistic characteristics of the response of structure and its control system in the frequency domain are analyzed in theory. Secondly, the structural responses of wind load under three conditions, including standard operating condition, cutting condition and limiting condition, are analyzed by the spectral analysis method in practice and the control effect of the damper on the structure is discussed. The results show that the tower structure has different response characteristics under different wind speeds, and the maximum displacement and acceleration of the structure are greatly reduced after the suspension tuned mass damper is set up.

Keywords: wind-power tower structure; spectrum analysis; damper. 